\title{
Situación ocupacional de los jóvenes egresados de la educación media: comparación entre los egresados de la formación técnico-profesional y la humanista-científica
}

\author{
Employment situation of young graduates of secondary education: a comparison between \\ the graduates of technical-professional training and humanistic-scientific training
}

\author{
Situação empregatícia dos jovens egressos do ensino médio: uma comparação \\ entre egressos do ensino técnico e humanístico-científico
}

\section{Iván Ortiz}

Universidad Alberto Hurtado, 2-8897147, iortiz@cide.cl

\begin{abstract}
RESUMEN
Se comparó la situación laboral de los jóvenes de 18 a 24 años egresados de la educación media técnico-profesional y humanista-científica de ambos sexos en relación a su situación ocupacional, a la formalidad contractual y a los ingresos de aquellos que se encuentran ocupados. Además, se comparó la situación laboral de estos jóvenes antes y después de la reforma de la educación media en 1998. En general, los egresados de la formación técnicoprofesional presentan leves ventajas laborales respecto de sus pares egresados de la formación humanista-científica. Pero más importantes son las diferencias de género: las mejores condiciones laborales las tienen los egresados hombres TP, luego los hombres HC, las mujeres TP y finalmente las mujeres HC. Este estudio muestra evidencia de que la reforma de la educación media técnico-profesional no significó un deterioro en el destino laboral de sus egresados, pese a haberse reducido el tiempo de duración de la formación en dos años.
\end{abstract}

Palabras clave: educación media, egresados, situación laboral, reforma educativa.

\begin{abstract}
It was compared the employment status of young people, aged 18 to 24 , graduated from technical-vocational secondary education and humanistic-scientific of both sexes, in relation to their employment situation, the formal contract and the income of those who are employed. It was also compared the employment status of these young people before and after the reform of the secondary education in 1998. In general, graduates of technical and vocational training have slight advantages in respect of graduates of humanistic and scientific training. But more important are gender differences: male graduates have better working conditions than female graduates. This study shows evidence that the reform of technical and vocational secondary education did not mean a deterioration in the employment prospects of graduates, although they have reduced the duration of training in two years.
\end{abstract}

Key words: secondary education, graduates, employment status, education reform.

\section{RESUMO}

Comparou-se a situação empregatícia de jovens de ambos os sexos, com idade entre 18 e 24, egressos do Ensino Médio em cursos técnicos e humanístico-científico, em relação à formalidade contratual e à renda dos empregados. Também comparou-se a situação empregatícia de jovens antes e depois da reforma do ensino médio, em 1998. Em geral, os concluintes do ensino técnico têm leves vantagens em relação aos concluintes da formação humanística-científica. O mais importante é a diferença de gênero, já que concluintes do sexo masculino do ensino técnico têm melhores condições de trabalho do que os concluintes do sexo masculino do humanísticocientífico e, depois dos concluintes e na mesma ordem, estão as concluintes. Este estudo evidencia que a reforma do ensino técnico não significou uma deterioração das perspectivas de emprego dos egressos, embora o tempo da formação tenha sofrido redução de dois anos.

Palavras chave: ensino médio, egressos, situação empregatícia, reforma educacional. 


\section{INTRODUCCIÓN}

En los últimos tres años, la Educación Media Técnico-Profesional ${ }^{1}$ en nuestro país ha sido objeto de varios análisis e investigaciones, que expresan la preocupación de diversos actores educativos en torno a su calidad y pertinencia (por ejemplo, Colegio de Profesores de Chile, 2007; Espinosa, 2008; Centro de Investigación y Desarrollo de la Educación, 2009; Ortiz, 2009). De ellos se desprende un diagnóstico sombrío respecto de varias dimensiones de su calidad, tales como logros deficientes, docentes sin formación idónea, falta de pertinencia de las actividades de aprendizaje, insuficiencia u obsolescencia de equipamiento e infraestructura, y otros. Pese a los problemas, se trata de una modalidad educativa con alta demanda, pues el $45 \%$ de los alumnos de $3^{\circ}$ y $4^{\circ}$ medio del país están matriculados en ella desde hace varios años.

Como se sabe, la EMTP experimentó un proceso de reforma en 1998, en el contexto de un proceso de revisión de la educación media, la cual a su vez era resultado de una preocupación mayor de la política educacional por los problemas de calidad y equidad del sector (Cox, 2003). La reforma construyó una lectura de la situación de la EMTP que puso el acento en dos grandes problemas: oferta desmedida de especialidades técnicas y duración excesiva de la formación técnica (Miranda, 2003). El primero de ellos consistía en el crecimiento exagerado e inorgánico de especialidades técnicas que se ofrecían a los alumnos, desconectadas del mundo productivo y sus requerimientos. La reforma redujo y actualizó sustancialmente el número de especialidades y definió sus perfiles de egreso en cooperación con el mundo productivo para asegurar su pertinencia.

El segundo problema se refería a la relación entre formación general y formación especializada que es razonable ofrecer a los alumnos que optan por una inserción laboral temprana, frente a lo cual la reforma innovó en forma decisiva postergando en dos años el inicio de la formación especializada, de $1^{\circ}$ a $3^{\circ}$ año medio, y disminuyendo su duración a dos años. De este modo, se alineaba con la discusión académica y las políticas a nivel internacional, las cuales recomendaban profundizar las competencias generales. (Ministerio de Educación, 1998). Cabe preguntarse qué impacto ha tenido esta medida radical en la calidad de la formación técnico-profesional.

Un estudio reciente revela que la principal crítica a la reforma de la EMTP, proveniente de los propios directivos y docentes de esta modalidad educativa, apunta a la disminución de los años de formación diferenciada destinados a la adquisición de competencias en las respectivas especialidades, cuestionándose la posibilidad de adquisición de estas competencias en tan poco tiempo e incidiendo, por tanto, en debilidades de la base formativa de los estudiantes egresados de este sistema (Sepúlveda, 2009). Pero no hay evidencias que confirmen o contradigan esta opinión.

Con las medidas antes señaladas, la reforma intentó modernizar la oferta educativa de la EMTP, pero no puso en duda la pertinencia social de esta modalidad educativa; por el contrario, reafirmó su justificación histórica en torno al trabajo: “...se ofrecerá a los alumnos y las alumnas oportunidades de realizar aprendizajes en un campo de especialización que facilite su acceso a un primer trabajo remunerado, atendiendo a sus intereses, aptitudes y disposiciones vocacionales, mediante una formación técnica en el

1 Nos referiremos a ella como EMTP o TP; y a la modalidad humanista-científica como EMHC o HC. 
ámbito de un sector del mundo productivo, que los prepare en forma efectiva para el trabajo y para responder con flexibilidad a la velocidad de los cambios tecnológicos" (Ministerio de Educación, 1998). Dadas las transformaciones en el sector productivo experimentadas por la sociedad desde la reforma anterior, la nueva propuesta cambió el enfoque formativo desde la preparación para un puesto de trabajo al desarrollo de competencias más amplias y flexibles para desenvolverse en un sector productivo. Insistió además en que la diferenciación técnico-profesional también habilita al estudiante para continuar estudios superiores y, por otra parte, planteó que es el conjunto de la experiencia de educación media el que posibilita alcanzar las competencias que permiten acceder y desarrollarse en el medio laboral, por cuanto la formación general tiene en ello un peso muy importante (Miranda, 2003).

¿Hasta qué punto un egresado de educación media TP está en mejores condiciones que un egresado HC para ingresar y permanecer en el mercado del trabajo? La disminución del número de años de formación diferenciada, ¿significó una merma en la eficacia ocupacional de la EMTP? Estas son las dos preguntas que orientan la presente investigación.

\section{REVISIÓN DE LA LITERATURA}

Un estudio encargado por el Ministerio de Educación (Arzola y otros, 1993) encontró que durante los dos años y medio consecutivos al egreso de la educación media en 1989, el porcentaje de ex-alumnos TP con empleo había fluctuado entre 54 y 75 (sin especificar si el empleo se relacionaba o no con la especialidad estudiada), obteniendo ingresos bajos pero levemente superiores a los que obtenían los egresados de la modalidad HC. Esto es, los egresados TP se ubicaban en los tramos superiores a $\$ 50.000$ de la época en mayor proporción que sus pares HC: $55 \%$ versus $43 \%$. Este estudio analizó también datos de ingresos de egresados de la enseñanza media provenientes de la encuesta CASEN de 1990, encontrando que el $67 \%$ de los $\mathrm{HC}$ y el $65 \%$ de los TP se ubicaba en los tramos de ingresos inferiores a $\$ 50.000$ de la época. Al mismo tiempo, encontraba que la polarización de los HC era mayor, pues hay más de ellos que ganan más y más de ellos que ganan menos que los TP. Estos hallazgos llevaron a concluir a los investigadores que ambos tipos de egresados constituían "mano de obra barata para el mercado laboral" (p. 35).

Cáceres y Bobenrieth (1993), encontraron también un patrón levemente favorable a los egresados TP en comparación con los HC, y añaden un dato interesante: trabajar en la especialidad estudiada aporta un diferencial salarial positivo con respecto a los egresados TP que no lo hacen ${ }^{2}$. Este estudio y el anterior sugieren que al comienzo de la década de los 90, el mercado tendía a reconocer la mejor formación para el trabajo de los egresados TP, pero en una magnitud tan poco significativa que permitía abrigar dudas acerca de la real eficiencia y relevancia de esta modalidad educativa.

Otro estudio (Bravo et al. 1998) aporta cifras de la situación laboral en 1997 de los egresados TP de 1991. Allí se revela que el $80 \%$ de los jóvenes se encontraba con empleo,

2 Estudio citado por Bravo y otros (1998). Estos resultados sugieren que los indicadores de eficacia de la TP deberían considerar la tasa de ocupados en la especialidad estudiada como un criterio más pertinente que la tasa total de ocupados. 
pero solo el $44 \%$ lo hacía en la especialidad que estudió. Por otra parte, el $30 \%$ de los egresados había realizado o estaba realizando estudios superiores técnicos o universitarios. Un aporte de este estudio es la comparación entre la situación de los egresados de las distintas modalidades de administración de los establecimientos TP, encontrando que no hay diferencias significativas entre ellas, tanto en inserción ocupacional como en el nivel de remuneraciones; en cambio, el costo fiscal por alumno era mayor en los establecimientos corporativizados en comparación con los municipalizados y particulares subvencionados.

Contreras et al. (2004) estudiaron las dinámicas de entrada y salida de la pobreza de las familias, siguiendo a la muestra de hogares de la encuesta Panel Casen entre 1996 y 2001. Encontraron que la educación técnica sirve como recurso para salir de la condición de pobreza pero no para evitar entrar en ella, pero lamentablemente su concepto de educación técnica no distingue entre la formación técnica secundaria y la superior, integrándolas a ambas en la misma noción.

Vargas et al. (2006) averiguaron el destino laboral de una muestra de 258 egresados TP del sector Administración y Comercio en la región metropolitana. Un año y medio después del egreso, solo el $36 \%$ estaba trabajando, de los cuales la mitad aproximadamente lo hacía en ocupaciones relacionadas con la especialidad estudiada.

Servat (2007) indagó el destino de una muestra de 1.234 egresados TP de la promoción 2004, de todas las familias ocupacionales en la región metropolitana. Si bien este estudio tampoco compara el destino de los egresados TP y HC, arroja alguna luz de la situación posterior a la reforma: el 50,7\% se encontraba trabajando al año siguiente del egreso, pero solo el $30,5 \%$ del total de egresados en la especialidad estudiada. Por otra parte, el $28,5 \%$ se encontraba estudiando (algunos de ellos estudiando y trabajando), aunque la mayor parte de ellos lo hacía en otra disciplina. En cualquier caso, la efectividad neta de la formación TP, entendida como su capacidad de preparar a los alumnos para ingresar a un trabajo y/o continuar estudios relacionados con la especialidad estudiada, alcanza en esta muestra solo al $38 \%$ de los egresados.

Sánchez y Escudero (2008) analizaron las trayectorias laborales y educacionales de los egresados de la enseñanza media a través de la Encuesta Panel CASEN, la cual permite hacer un seguimiento a una muestra de los encuestados por la Encuesta CASEN en 1996, 2001 y 2006. Hallaron que el 19\% de los egresados de la EMHC en 1996, se encontraban trabajando cinco años después, en el 2001; en cambio la gran mayoría del resto se encontraba estudiando. Al año 2006, el 60\% de la cohorte de egresados de 1996 se encontraba trabajando, la gran mayoría de ellos como profesionales universitarios. Por otra parte, de los egresados de la EMTP en 1996, el 82\% se encontraba trabajando cinco años después, porcentaje que aumenta a $91 \%$ en el 2006.

Sevilla et al. (2009) siguieron el destino laboral y educacional de una muestra de 4200 egresados en el 2003 de la EMTP y de 1000 egresados en el mismo año de la modalidad humanista-científica, por un periodo de cinco años. Encontraron que los egresados de la EMTP en comparación con sus pares egresados de la EMHC tienen un porcentaje mayor de ocupados y menor de desocupados, y mejores indicadores de formalidad en el contrato de trabajo. En cuanto a los ingresos, se encontraron importantes diferencias de acuerdo al tipo de especialidad de la formación TP. Así, de los cuatro sectores de especialidad estudiados, los egresados del sector de Administración y Comercio, son los que registran los ingresos más bajos, incluso inferiores a los que reportan los egresados de la EMHC (excepto la especialidad de contabilidad); los egresados del sector Metalmecánica reportan ingresos que fluctúan 
entre el 16\% y el 32\% más que el de los egresados de la EMHC, según sea la especialidad de este sector; los egresados del sector Minero son los que reportan los mayores ingresos, superando en un $48 \%$ al de los egresados de la EMHC; finalmente, los egresados del sector Agropecuario también reportan ingresos más bajos que sus pares de la EMHC.

El estudio anterior comparó también el destino educacional de los egresados de ambas modalidades, encontrando una situación bastante más favorable en los egresados de la EMHC, como era de esperarse: $72 \%$ de ellos reporta seguir o haber seguido estudios superiores en algún momento del período estudiado, contra un $47 \%$ de sus pares de la EMTP. En nuestra opinión, la continuidad de estudios superiores hace que los indicadores de empleo de ambas poblaciones estudiadas no sean del todo comparables, puesto que la modalidad HC, a diferencia de la TP, no se orienta hacia el trabajo. Por ello, sería interesante comparar a aquellos egresados de ambas modalidades que no siguen estudios superiores. Pese al aumento de la cobertura de la educación superior, la interrupción de los estudios al finalizar la educación media sigue siendo la situación de la mayoría de los jóvenes, especialmente de aquellos provenientes de los quintiles inferiores de ingreso; se trataría de ver si, justamente, la EMTP constituye o no una ventaja para esta población.

Por otra parte, ninguno de los estudios antes citados se refiere a la situación de género, es decir a las diferencias eventuales del destino laboral de los egresados de la educación media de ambos sexos. Un informe reciente sobre educación superior en Chile (OCDE-Banco Mundial, 2009) denuncia que las mujeres están sub-representadas en la educación superior en relación a su participación en la matrícula de enseñanza media, y obtienen menos puntaje que los hombres en la prueba PSU. Por tanto, es de interés averiguar si, además, el mercado laboral discrimina de alguna forma entre los egresados hombres y mujeres de la educación media que buscan trabajo.

No existe ningún estudio nacional que describa, compare o compruebe el eventual impacto de la reducción del tiempo destinado a la formación técnica y el aumento en dos años de la formación general. A nivel internacional, se encuentran muy pocos estudios del impacto de la educación secundaria vocacional. Una revisión reciente (Jakubowski y otros, 2010) encuentra evidencia de que la mayoría de los egresados de este tipo de educación, en varios países, siguen estudios superiores más que entrar en ocupaciones manuales. No obstante, estos autores probaron que una reforma similar a la chilena, en Polonia, que acortó los años de duración de la formación secundaria vocacional, tuvo como efecto mejorar la formación general de los estudiantes y aumentar significativamente sus resultados de aprendizaje en las pruebas PISA en 2006 de lectura, matemática y ciencias, respecto de las mismas pruebas PISA en 2001.

\section{METODOLOGÍA}

Los objetivos específicos del estudio son los siguientes:

1. Comparar la situación laboral actual de los egresados de la educación media TP y HC de ambos sexos, en relación a su situación ocupacional y a la formalidad contractual y los ingresos de aquellos que se encuentran ocupados.

2. Comparar la situación laboral de los egresados de la educación media TP y HC de ambos sexos, antes y después de la reforma. 
Los datos del estudio provienen de la Encuesta de Caracterización Socio-económica Nacional (CASEN) que se aplica periódicamente a una muestra representativa de la población en hogares particulares, con cobertura nacional. Esta encuesta recoge, entre muchos otros, datos de la situación ocupacional de los integrantes de los hogares encuestados, el tipo de ocupación, la tenencia y tipo de contrato de trabajo, la situación previsional y los ingresos del trabajo, que constituyen las variables en estudio.

De acuerdo al objetivo 1, se analizan los datos de la encuesta CASEN 2009. Se estudia a las personas entre 18 y 24 años que: i) declararon no estar estudiando en el momento de ser encuestados, y ii) haber cursado la enseñanza media completa (TP o HC) como últimos estudios realizados. Cabe señalar que los tipos de educación definidos en la encuesta CASEN permiten distinguir entre educación media TP y HC, pero no permiten diferenciar los subtipos de especialidades TP cursados por los egresados.

De acuerdo al objetivo 2, se comparan los datos anteriores de la encuesta CASEN 2009 con datos paralelos de la CASEN 2000. Es decir, se estudia a las personas entre 18 y 24 años que declararon no estar estudiando en el momento de ser encuestados, y haber cursado la enseñanza media completa (TP o HC) como últimos estudios realizados. En el año 2000, los egresados que tenían 18 a 24 años habían estudiado de acuerdo al currículo previo a la reforma, por lo tanto los ex alumnos de la modalidad técnico-profesional hicieron 4 o 5 años de estudios técnicos. En el año 2009, los egresados de ese grupo de edad fueron formados en el currículo de la reforma, con dos años de formación especializada en la modalidad técnica. Egresados de edades superiores, 26 años y más, no alcanzaron a formarse en el currículo de la reforma.

Se trata de un estudio descriptivo que compara frecuencias en tablas de contingencia, y promedios (de ingresos), mediante el Statistical Package for Social Sciences (SPSS). Obviamente no se trata de un estudio de seguimiento de cohortes, donde los sujetos se pueden comparar consigo mismos a través del tiempo. En nuestro caso, se comparan grupos de individuos distintos en los años estudiados; la comparabilidad se basa en el análisis de la estructura de las diferencias entre los egresados TP y HC, ¿cambia esa estructura de diferencias en el 2009 con respecto del 2000?

\section{RESULTADOS}

Un total de 8.214 sujetos de la CASEN 2000 y de 11.338 sujetos de la CASEN 2009 cumplen las condiciones estipuladas más arriba; sobre estos totales se hicieron los análisis estadísticos. En el 2000, 63\% del total mencionado son egresados HC y $37 \%$ son TP; en el $2009,66 \%$ son HC y $34 \%$ son TP. La composición por sexo es equilibrada en ambos años, con una ligera preponderancia de los hombres entre los egresados TP encuestados el 2000.

\subsection{ANÁLISIS GENERAL}

En ambos años, la mayoría de los egresados de la educación media en estudio viven en hogares donde asumen el rol de hijos o hijastros. Aquellos que son jefe de núcleo familiar o su pareja, fluctúan entre $30 \%$ y $34 \%$. La distribución de roles es muy similar en ambos años para los egresados de ambas modalidades. 
Roles en el hogar de los sujetos

\begin{tabular}{|l|c|c|c|c|}
\cline { 2 - 5 } \multicolumn{1}{c|}{} & \multicolumn{2}{c|}{ CASEN 2000 } & \multicolumn{2}{c|}{ CASEN 2009 } \\
\cline { 2 - 5 } \multicolumn{1}{c|}{} & Egresados HC & Egresados TP & Egresados HC & Egresados TP \\
\hline Jefe(a) de núcleo & $19,1 \%$ & $18,6 \%$ & $18,1 \%$ & $19,0 \%$ \\
\hline Esposo(a)/pareja & $15,7 \%$ & $13,9 \%$ & $12,2 \%$ & $11,1 \%$ \\
\hline Hijo(a), hijastro(a) & $59,4 \%$ & $62,6 \%$ & $62,4 \%$ & $63,6 \%$ \\
\hline Otro familiar & $5,4 \%$ & $4,8 \%$ & $6,8 \%$ & $5,8 \%$ \\
\hline No familiar & $0,5 \%$ & $0,1 \%$ &, $6 \%$ &, $4 \%$ \\
\hline Total & $100 \%$ & $100 \%$ & $100 \%$ & $100 \%$ \\
\hline
\end{tabular}

La distribución de sujetos que viven en hogares en situación de pobreza es relativamente similar para los egresados TP y HC. No obstante, se observa que los egresados HC pobres suman el 15,8\% versus el 18,5\% de los TP en el 2000. En cambio en el 2009 los $\mathrm{HC}$ pobres suben a 17,3\% y los TP bajan a 16,3\%. Pese a que las diferencias son leves, los hogares donde viven los egresados TP mejoran su relación con la pobreza en el 2009, en vez de empeorar.

Situación de pobreza de los hogares de los egresados

\begin{tabular}{|l|c|c|c|c|}
\cline { 2 - 5 } \multicolumn{1}{c|}{} & \multicolumn{2}{c|}{ CASEN 2000 } & \multicolumn{2}{c|}{ CASEN 2009 } \\
\cline { 2 - 5 } \multicolumn{1}{c|}{} & Egresados HC & Egresados TP & Egresados HC & Egresados TP \\
\hline Indigente & $2,8 \%$ & $4,2 \%$ & $4,0 \%$ & $4,2 \%$ \\
\hline Pobre no indigente & $13,0 \%$ & $14,3 \%$ & $13,3 \%$ & $12,1 \%$ \\
\hline No pobre & $84,2 \%$ & $81,4 \%$ & $82,7 \%$ & $83,7 \%$ \\
\hline Total & $100 \%$ & $100 \%$ & $100,0 \%$ & $100,0 \%$ \\
\hline
\end{tabular}

Se observan diferencias significativas en la condición de actividad entre los egresados de ambas modalidades, tanto en el 2000 como en el 2009³: Los TP presentan alrededor de 8 puntos porcentuales más de ocupados que los HC. En cambio, la proporción de desocupados es similar en ambos grupos educacionales. Este patrón de diferencias es similar en el 2000 en cuanto al porcentaje de ocupados; sin embargo, la situación de los desocupados es algo más favorable a los egresados HC en ese año, pues aumentan dos puntos porcentuales en el 2009, al paso que los TP desocupados bajan muy levemente. Es decir, los HC empeoran, no así los TP.

3 Prueba de Chi cuadrado significativa a probabilidad ,000 en ambos años. 
Condición de actividad de los egresados TP y HC

\begin{tabular}{|l|c|c|c|c|}
\cline { 2 - 5 } \multicolumn{1}{c|}{} & \multicolumn{2}{c|}{ CASEN 2000 } & \multicolumn{2}{c|}{ CASEN 2009 } \\
\cline { 2 - 5 } \multicolumn{1}{c|}{} & Egresados HC & Egresados TP & Egresados HC & Egresados TP \\
\hline Ocupados & $50,9 \%$ & $59,5 \%$ & $47,9 \%$ & $56,0 \%$ \\
\hline Desocupados & $12,8 \%$ & $16,9 \%$ & $14,9 \%$ & $15,3 \%$ \\
\hline Inactivos & $36,3 \%$ & $23,6 \%$ & $37,1 \%$ & $28,6 \%$ \\
\hline Total & $100 \%$ & $100 \%$ & $100 \%$ & $100 \%$ \\
\hline
\end{tabular}

Las similares cifras de desocupados que se observan en el 2009 sugieren que la mayor proporción de ocupados que presentan los egresados TP no se debería a una preferencia del mercado ocupacional por ellos, sino a su mayor disposición a trabajar, por cuanto este grupo presenta una proporción bastante menor de inactivos que los egresados HC.

La población en estudio que se encuentra ocupada en el 2009 se concentra en oficios tales como "trabajadores de los servicios y vendedores de comercio y mercados", "trabajadores no calificados", "oficiales, operarios y artesanos de artes mecánicas y otros oficios" y "empleados de oficina". Esta distribución es bastante similar entre egresados de la formación HC y TP. Sin embargo, hay algunas diferencias interesantes": hay más trabajadores no calificados entre los egresados HC que entre los TP; y hay más oficiales, operarios y artesanos entre los TP que entre los $\mathrm{HC}$.

Oficios desempeñados por los egresados HC y TP que se encuentran ocupados

\begin{tabular}{|l|r|r|r|r|}
\cline { 2 - 5 } \multicolumn{1}{c|}{} & \multicolumn{2}{|c|}{ CASEN 2000} & \multicolumn{2}{c|}{ CASEN 2009} \\
\cline { 2 - 5 } \multicolumn{1}{c|}{} & HC & TP & HC & \multicolumn{1}{c|}{ TP } \\
\hline Fuerzas armadas &, $5 \%$ &, $4 \%$ &, $6 \%$ &, $4 \%$ \\
\hline Miembros del Poder Ejecutivo y de los cuerpos legislativos & $1,3 \%$ & $1,0 \%$ &, $5 \%$ &, $5 \%$ \\
\hline Profesionales, científicos e intelectuales &, $9 \%$ & $2,4 \%$ &, $1 \%$ &, $1 \%$ \\
\hline Técnicos y profesionales de nivel medio & $4,9 \%$ & $9,5 \%$ & $4,5 \%$ & $15,1 \%$ \\
\hline Empleados de oficina & $17,1 \%$ & $28,8 \%$ & $9,8 \%$ & $12,7 \%$ \\
\hline Trabajadores de los servicios y vendedores de comercio y mercados & $30,3 \%$ & $21,1 \%$ & $23,3 \%$ & $18,1 \%$ \\
\hline Agricultores y trabajadores calificados agropecuarios y pesqueros & $3,1 \%$ & $2,4 \%$ & $4,6 \%$ & $4,4 \%$ \\
\hline Oficiales, operarios y artesanos de artes mecánicas y otros oficios & $13,1 \%$ & $17,6 \%$ & $12,7 \%$ & $18,3 \%$ \\
\hline Operadores y montadores de instalaciones y maquinaria & $6,7 \%$ & $6,5 \%$ & $9,1 \%$ & $6,8 \%$ \\
\hline Trabajadores no calificados & $22,2 \%$ & $10,1 \%$ & $34,4 \%$ & $23,4 \%$ \\
\hline Ocupación no bien especificada &, $1 \%$ &, $1 \%$ &, $2 \%$ &, $2 \%$ \\
\hline Total & $100,0 \%$ & $100,0 \%$ & $100 \%$ & $100 \%$ \\
\hline
\end{tabular}

4 Prueba de Chi cuadrado significativa a probabilidad ,000. 
De la diversidad de oficios desempeñados por los egresados HC y TP, aquellos que aportan indicios de cambios entre el 2000 y el 2009 son los siguientes. Técnicos y profesionales de nivel medio: los egresados TP en estos oficios aumentaron unos cinco puntos porcentuales en el 2009 respecto del 2000, en cambio los HC se mantuvieron. Empleados de oficina: todos disminuyen en el 2009, pero los TP disminuyen bastante más que los HC. Trabajadores de los servicios y vendedores de comercio: todos disminuyen, pero los egresados HC disminuyen más que los TP. Trabajadores no calificados: todos aumentan significativamente en el 2009 , en la misma proporción.

Las diferencias entre el 2000 y el 2009 no presentan un patrón claro. Si bien en el 2009 los egresados TP son más reconocidos como técnicos o profesionales de nivel medio que en el 2000, también aumentan los que son clasificados como trabajadores no calificados. Al parecer, muchos egresados TP que en el 2000 eran clasificados como empleados de oficina, en el 2009 los son como trabajadores no calificados. Es posible que la crisis económica globalizada del 2009 explique esta suerte de precarización del empleo, pero también es posible que estos datos reflejen un eventual ajuste de los criterios de clasificación por parte de los encuestadores entre ambos años. En todo caso, tanto en el 2000 como en el 2009 los egresados TP que son clasificados como trabajadores no calificados son menos numerosos que sus pares HC; esto podría indicar que el título técnico del cual son o se sienten portadores los egresados TP, les permitiría cierta protección en cuanto a la clase de ocupación que logran encontrar: más calificada, aunque no necesariamente mejor remunerada.

En el 2009, la gran mayoría de quienes se encuentran ocupados tiene un contrato firmado de trabajo; los egresados TP presentan un porcentaje levemente superior a los $\mathrm{HC}$ en tenencia de contrato firmado 5 .

Tenencia de contrato de trabajo de los que se encuentran ocupados

\begin{tabular}{|l|c|c|c|c|}
\cline { 2 - 5 } \multicolumn{1}{c|}{} & \multicolumn{2}{c|}{ CASEN 2000 } & \multicolumn{2}{c|}{ CASEN 2009 } \\
\cline { 2 - 5 } \multicolumn{1}{c|}{} & $\begin{array}{c}\text { Egresados } \\
\text { HC }\end{array}$ & $\begin{array}{c}\text { Egresados } \\
\text { TP }\end{array}$ & $\begin{array}{c}\text { Egresados } \\
\text { HC }\end{array}$ & $\begin{array}{c}\text { Egresados } \\
\text { TP }\end{array}$ \\
\hline Sí, firmó & - & - & $73,7 \%$ & $77,3 \%$ \\
\hline Si, pero no ha firmado & - & - & $2,3 \%$ & $2,0 \%$ \\
\hline No tiene o no ha firmado & $26,2 \%$ & $21,8 \%$ & $21,5 \%$ & $18,4 \%$ \\
\hline No se acuerda o no sabe si firmó contrato & $2,3 \%$ & $0,9 \%$ & $2,5 \%$ & $2,3 \%$ \\
\hline
\end{tabular}

Las preguntas de la encuesta CASEN sobre contrato de trabajo presentan diferencias entre ambos años; de hecho, en el 2000 el tema se aborda con una sola pregunta, en cambio en el 2009 con dos. De modo que solo se presentan los datos de las categorías de respuesta que son comparables. Se observa una disminución, entre el 2000 y el 2009

5 Prueba de Chi cuadrado significativa a probabilidad ,032. 
de aquellos ocupados que no tienen o no han firmado un contrato de trabajo. Si bien la disminución es más acentuada en los $\mathrm{HC}$ que en los TP, estos últimos superan a los primeros en tenencia de contrato en ambos años (esto, implícito en los datos del 2000).

En cuanto a la situación previsional, también hay algunas diferencias en las preguntas de la encuesta en ambos años, por lo que se presentan a continuación las categorías de respuesta comparables. La mayoría de los egresados de la educación media se encuentra cotizando en algún sistema previsional, tanto en el 2000 como en el 2009. En ambos años, los egresados TP que cotizan superan a los $\mathrm{HC}$ en algunos puntos porcentuales ${ }^{6}$. No hay mayores diferencias en la situación previsional de los grupos en estudio entre el 2000 y el 2009: ambos grupos mejoran sus porcentajes de afiliación.

Afiliación a institución previsional de los egresados HC y TP que se encuentran ocupados

\begin{tabular}{|l|c|c|c|c|}
\cline { 2 - 5 } \multicolumn{1}{c|}{} & \multicolumn{2}{c|}{ CASEN 2000 } & \multicolumn{2}{c|}{ CASEN 2009 } \\
\cline { 2 - 5 } \multicolumn{1}{c|}{} & $\begin{array}{c}\text { Egresados } \\
\text { HC }\end{array}$ & $\begin{array}{c}\text { Egresados } \\
\text { TP }\end{array}$ & $\begin{array}{c}\text { Egresados } \\
\text { HC }\end{array}$ & $\begin{array}{c}\text { Egresados } \\
\text { TP }\end{array}$ \\
\hline $\begin{array}{l}\text { Si, AFP } \\
\text { (Administradora de Fondos de Pensiones) }\end{array}$ & $60,4 \%$ & $66,3 \%$ & $67,9 \%$ & $72,8 \%$ \\
\hline No está afiliado & $36,8 \%$ & $30,7 \%$ & $26,3 \%$ & $22,3 \%$ \\
\hline No sabe/Sin dato & $0,3 \%$ & $0,2 \%$ & $3,4 \%$ & $3,3 \%$ \\
\hline
\end{tabular}

A continuación se analizan dos tipos de ingresos de los sujetos de la muestra: los percibidos en la ocupación principal y los ingresos totales provenientes de su trabajo, es decir, los que provienen de su ocupación principal más los de otras que pudieran tener.

Puesto que los montos de ingreso no son comparables en el tiempo y no han sido ajustados, lo que se compara es la estructura de las diferencias entre ambos tipos de egresados en los dos años.

En 2009, la media del ingreso mensual percibido por la ocupación principal de los egresados TP es superior en unos $\$ 5.000$ al de sus pares HC, diferencia que no es estadísticamente significativa. Esta situación favorable a los egresados TP se amplifica para el caso del ingreso mensual del trabajo. ${ }^{7}$

Se aprecia que tanto en el 2000 como en el 2009, los egresados TP tienen ingresos superiores a sus pares HC, en promedio. Pero mientras en el 2000 los primeros ganan un 9,1\% más en su ocupación principal que los segundos, en el 2009 esa cifra desciende a 2,6\%. En el caso de los ingresos totales provenientes del trabajo, las diferencias entre ambos grupos son favorables a los TP en un 9,3\% en el 2000 , y en $5,7 \%$ en el 2009.

$6 \quad$ Prueba de Chi cuadrado significativa a probabilidad ,007 para el año 2009.

7 Prueba T significativa a probabilidad ,003. 
Ingresos del trabajo de los egresados TP y HC que se encuentran ocupados (pesos)

\begin{tabular}{|l|c|c|c|c|}
\cline { 2 - 5 } \multicolumn{1}{c|}{} & \multicolumn{2}{c|}{ CASEN 2000 } & \multicolumn{2}{c|}{ CASEN 2009 } \\
\cline { 2 - 5 } \multicolumn{1}{c|}{} & Egresados HC & Egresados TP & Egresados HC & Egresados TP \\
\hline De la ocupación principal & 131.589 & 143.621 & 199.892 & 205.059 \\
\hline Desviación típica & 96.317 & 89.658 & 97.621 & 130.708 \\
\hline Del trabajo en total & 134.743 & 147.375 & 213.579 & 225.953 \\
\hline Desviación típica & 99.012 & 136.964 & 131.121 & 180.771 \\
\hline
\end{tabular}

Los ingresos exhibidos no están corregidos según la jornada trabajada, pues la gran mayoría de ocupados en ambos grupos tiene jornada completa (85\%). Los egresados TP presentan una ligera mayor proporción de trabajadores con jornada completa, inferior a los dos puntos porcentuales, la que no lograría explicar las diferencias de ingreso.

Puede apreciarse que se trata de ingresos modestos en todos los casos. Considérese que el salario mínimo en el 2000 era de $\$ 100.000$, y en el 2009 , de $\$ 165.000$, en pesos de cada año.

El cuadro siguiente compara los porcentajes de individuos que ganan más o menos del salario mínimo en ambos años. No se observan diferencias entre ambos tipos de egresados en el año 2009, en cambio en el 2000 la situación es favorable a los egresados TP.

Relación del ingreso de la ocupación principal con el salario mínimo

\begin{tabular}{|l|c|c|c|c|}
\cline { 2 - 5 } \multicolumn{1}{c|}{} & \multicolumn{2}{c|}{ CASEN 2000 } & \multicolumn{2}{c|}{ CASEN 2009 } \\
\cline { 2 - 5 } \multicolumn{1}{c|}{} & Egresados HC & Egresados TP & Egresados HC & Egresados TP \\
\hline Salario mínimo o menos & $42,4 \%$ & $36,1 \%$ & $25,5 \%$ & $24,8 \%$ \\
\hline Más del salario mínimo & $57,6 \%$ & $63,9 \%$ & $74,5 \%$ & $75,2 \%$ \\
\hline Total & $100,0 \%$ & $100,0 \%$ & $100,0 \%$ & $100,0 \%$ \\
\hline
\end{tabular}

\subsection{ANÁLISIS POR SEXO}

Los resultados anteriores fueron analizados según el sexo de los encuestados. Se observan diferencias entre hombres y mujeres para varios indicadores de la situación laboral.

En 2009, hay más mujeres que hombres inactivos, y más hombres que mujeres ocupados, en ambas modalidades de egreso. Pese a que los egresados TP superan a los HC de su mismo género en el porcentaje de ocupados, la situación ocupacional de los hombres HC es mejor que la de las mujeres TP. Se observa también que las diferencias por sexo son más acentuadas que las diferencias por modalidad de estudio de los encuestados, y que tales diferencias no se refieren al número de desocupados. De modo que estos datos 
confirman que el mayor nivel de ocupación de los egresados TP no se debería a una preferencia del mercado sino a una menor disposición a trabajar por parte de los egresados $\mathrm{HC}$, y permiten precisar que las mujeres $\mathrm{HC}$ son las menos dispuestas a ello.

Condición de actividad de los egresados por sexo

\begin{tabular}{|l|c|c|c|c|}
\cline { 2 - 5 } \multicolumn{1}{c|}{} & \multicolumn{4}{c|}{ CASEN 2000 } \\
\cline { 2 - 5 } \multicolumn{1}{c|}{} & \multicolumn{2}{c|}{ HOMBRES } & \multicolumn{2}{c|}{ MUJERES } \\
\cline { 2 - 5 } \multicolumn{1}{c|}{} & H-C & T-P & H-C & T-P \\
\hline Ocupados & $62,1 \%$ & $65,6 \%$ & $41,2 \%$ & $53,7 \%$ \\
\hline Desocupados & $12,7 \%$ & $17,8 \%$ & $13,0 \%$ & $16,0 \%$ \\
\hline Inactivos & $25,2 \%$ & $16,6 \%$ & $45,9 \%$ & $30,3 \%$ \\
\hline Total & 100 & 100 & 100 & 100 \\
\hline
\end{tabular}

\begin{tabular}{|c|c|c|c|}
\hline \multicolumn{3}{|c|}{ CASEN 2009 } \\
\hline HOMBRES & \multicolumn{2}{c|}{ MUJERES } \\
\hline H-C & T-P & H-C & T-P \\
\hline $60,3 \%$ & $65,7 \%$ & $35,2 \%$ & $45,5 \%$ \\
\hline $15,4 \%$ & $15,6 \%$ & $14,5 \%$ & $15,0 \%$ \\
\hline $24,3 \%$ & $18,7 \%$ & $50,3 \%$ & $39,5 \%$ \\
\hline 100 & 100 & 100 & 100 \\
\hline
\end{tabular}

En el 2000, la condición de actividad de hombres y mujeres también es diferente: son más los hombres ocupados, y son más las mujeres inactivas ${ }^{8}$. En este marco, se observa una disminución evidente del porcentaje de mujeres ocupadas en el 2009 respecto del 2000 y, complementariamente, un aumento también evidente de mujeres inactivas, especialmente las mujeres TP. Los hombres ocupados también disminuyen pero levemente y solo los hombres HC, puesto que los hombres TP mantienen sus niveles de ocupación. En cuanto a la desocupación, las mujeres de ambas modalidades de estudio mantienen relativamente constantes sus niveles en los años observados. En cambio, los hombres pasan de una situación en el 2000 más bien desfavorable a los TP a una situación en el 2009 en que ambas modalidades se igualan. Tal vez lo más significativo de la comparación entre ambos momentos es el cambio de condición de actividad de muchas mujeres, de ocupadas a inactivas, lo cual podría deberse a la crisis económica del 2009, pues suele suceder que en periodos de escasez del empleo, las mujeres tienden a ceder sus posibilidades a los hombres.

En cuanto a los ingresos, los hombres presentan ingresos por el trabajo superiores a los de las mujeres. Estas diferencias podrían explicarse al menos en parte por la mayor proporción de mujeres que trabajan una jornada parcial: entre 7 y 8 puntos porcentuales más que los hombres. Por lo anterior, los datos de los cuadros siguientes excluyen del análisis a los sujetos que trabajan jornada parcial.

En el año 2000 se observa el mismo patrón de diferencias de ingreso entre hombres y mujeres que en el 2009, es decir, los hombres de ambas modalidades de estudio ganan más que sus pares mujeres. La jerarquía de ingresos es también la misma, encabezada por los hombres TP, luego los hombres HC, luego las mujeres TP y finalmente las mujeres HC. Asimismo, se observa también que las diferencias por sexo son más acentuadas que las diferencias por modalidad de estudio de los encuestados.

8 Las diferencias por sexo en ambos años presentan un Chi cuadrado significativo a p. ,000. 
Ingresos de la ocupación principal por sexo: promedio en pesos

\begin{tabular}{|l|l|c|c|}
\cline { 3 - 4 } \multicolumn{2}{c|}{} & CASEN 2000 & CASEN 2009 \\
\hline \multirow{2}{*}{ Educación Media Científico-Humanística } & Hombre & 130.752 & 210.518 \\
\cline { 2 - 4 } & Mujer & 121.470 & 185.489 \\
\hline \multirow{2}{*}{ Totacación Media Técnica Profesional } & Hombre & 148.905 & 215.643 \\
\cline { 2 - 4 } & Mujer & 128.162 & 188.843 \\
\hline & Hombre & 137.871 & 212.406 \\
\cline { 2 - 4 } & Mujer & 124.408 & 186.856 \\
\hline
\end{tabular}

Pero hay algunas diferencias entre ambos años. En el año 2000 las diferencias de ingreso entre los hombres TP y HC son más marcadas que en el 2009, pues en el 2000 los primeros ganan 13,8\% más que los segundos, y en el 2009 esa diferencia se reduce a un $2,4 \%$ más. En el caso de las mujeres, la diferencia a favor de las TP no es tan marcada en el 2000, y lo es aún menos en el $2009^{9}$.

En cuanto a los ingresos totales provenientes del trabajo, el patrón de diferencias entre hombres y mujeres es igual, en ambos años, al de los ingresos de la ocupación principal. Las diferencias por sexo son estadísticamente significativas. Se observa la misma jerarquía y la misma relación entre los hombres TP y HC, es decir, la diferencia entre sus ingresos totales es más marcada en el 2000 que en el 2009.

Ingresos totales del trabajo por sexo: promedios en pesos

\begin{tabular}{|l|l|c|c|}
\cline { 3 - 4 } \multicolumn{2}{c|}{} & CASEN 2000 & CASEN 2009 \\
\hline \multirow{2}{*}{ Educación Media Científico-Humanística } & Hombre & 136.532 & 231.544 \\
\cline { 2 - 4 } & Mujer & 124.474 & 201.755 \\
\hline \multirow{2}{*}{ Educación Media Técnica Profesional } & Hombre & 158.581 & 246.804 \\
\cline { 2 - 4 } & Mujer & 130.799 & 209.168 \\
\hline \multirow{2}{*}{ Total } & Hombre & 145.209 & 237.157 \\
\cline { 2 - 4 } & Mujer & 127.255 & 204.769 \\
\hline
\end{tabular}

Otros indicadores de la situación laboral de la población de ambos sexos que se encuentra ocupada también son favorables a los hombres. En el año 2009, el $16 \%$ de los hombres HC no tiene contrato de trabajo, versus el $22 \%$ de las mujeres HC; entre los egresados TP esa diferencia es de $15 \%$ versus $17 \%$ respectivamente. Por otra parte,

9 En el año 2000, las diferencias entre hombres y mujeres son estadísticamente significativas según la prueba $\mathrm{T}$ (probabilidad $=, 000$ ) y también lo son las diferencias entre hombres HC y TP y entre mujeres HC y TP. En el año 2009 solo son significativas las diferencias entre hombres y mujeres. 
el 19,7\% de los hombres HC no está cotizando en algún sistema previsional, versus el $34,7 \%$ de las mujeres HC; entre los egresados TP, esa diferencia es de 18,5 versus $27,5 \%$ respectivamente. Todas las diferencias anteriores son estadísticamente significativas, salvo la segunda $(15 \%-17 \%)^{10}$.

\section{CONCLUSIONES}

Se comparó la situación laboral actual de los jóvenes de 18 a 24 años egresados de la educación media TP y HC de ambos sexos, en relación a su situación ocupacional, a la formalidad contractual y a los ingresos de aquellos que se encuentran ocupados. Además, se comparó la situación laboral de los jóvenes egresados de la educación media TP y HC de ambos sexos, antes y después de la reforma de la EMTP. Las principales conclusiones son las siguientes.

En general, los egresados de la formación técnico-profesional presentan algunas ventajas respecto de sus pares egresados de la formación humanista-científica. Presentan actualmente una mayor tasa de ocupados, de ocupados con contrato y de ocupados cotizando en el sistema previsional; y mayores ingresos provenientes del trabajo, tanto de la ocupación principal como de otros trabajos secundarios. No obstante, llama la atención la escasa magnitud de las diferencias, pese a ser estadísticamente significativas.

Cabe preguntarse si esta diferencia a favor de los portadores de una certificación técnico-profesional se puede explicar por un reconocimiento de sus ventajas por parte del mercado laboral. Dados los hallazgos del estudio, cabe una duda razonable, por lo menos en lo que se refiere a la oportunidad de encontrar empleo si se lo busca. Al parecer, en las ventajas de los egresados TP influye su identificación más temprana con el mundo del trabajo, y su voluntad de participar en él, en comparación con sus pares $\mathrm{HC}$, quienes presentan mayor tendencia a la inactividad. Por otra parte, la revisión de la literatura nacional sugiere que el mercado laboral sí reconoce a los egresados TP, pero solo a algunos, por ejemplo, a los especializados en el área minera y metalmecánica.

De lo que no quedan dudas razonables, es que el mercado laboral trata mejor a los hombres que a las mujeres, pues a igual nivel de estudios, obtienen ingresos superiores por el trabajo y más formalidad de los contratos. El cruce de las variables género de los encuestados y modalidad de estudios secundarios, produce un orden jerárquico en que las mejores condiciones laborales las tienen los egresados hombres TP, luego los hombres $\mathrm{HC}$, las mujeres TP y finalmente las mujeres HC. En cuanto a los ingresos totales del trabajo, la diferencia entre el primero y el último sub-grupo es de unos $\$ 45.000$; es decir, los hombres egresados de la formación técnica ganan un $22 \%$ más que sus compañeras egresadas de la formación científico-humanista. En general, las diferencias por género son más grandes que las diferencias por modalidad de estudios.

Las pequeñas ventajas de los egresados TP sobre sus pares HC presentan estabilidad en el tiempo, pues la situación es similar en el 2000 y en el 2009. Este estudio muestra evidencia de que la reforma de la educación media técnico-profesional no significó un deterioro en el destino laboral de los egresados, pese a haberse reducido el tiempo de

10 Prueba de Chi cuadrado significativa a probabilidad ,000. 
duración de la formación en dos años. No obstante, la disminución en 2009 de la brecha de los ingresos medios de ambos grupos, en relación con el 2000, podría sugerir un deterioro relativo de los ingresos de los egresados TP. Pero se trata más bien de un mejoramiento de los ingresos de los egresados HC que puede ser explicado por el incremento sostenido del salario mínimo en el período estudiado (Marinakis, 2009). En efecto, el incremento del salario mínimo real tiende a favorecer a los que ganan menos, pues les sube el piso salarial, favoreciendo más en este caso a los egresados HC. Por otra parte, las situaciones de crisis económica, como la del año 2009, tienden a concentrar trabajadores en niveles cercanos al salario mínimo, frenando de este modo los ingresos relativamente más altos de los egresados TP. El año 2000, en cambio, encuentra a la economía del país recuperándose de la crisis asiática, con más trabajadores ganando ingresos superiores al salario mínimo en comparación con los años anteriores (Marinakis, 2005)

En el marco de estas conclusiones, es pertinente reflexionar sobre estos hechos desde las políticas públicas en educación. El Estado requiere estructurar un sistema escolar que asegure igualdad de oportunidades educativas para todos, discriminando positivamente a favor de la población socialmente desfavorecida, para aumentar sus posibilidades de movilidad social ascendente y de participación de los bienes materiales y culturales. Cabe preguntarse en qué medida y cómo la enseñanza media técnicoprofesional contribuye a ello. A la luz de los resultados de este estudio y de la revisión de otros, parece razonable considerar dos medidas complementarias. Por una parte, revisar la duración de la enseñanza de las especialidades técnicas y tratarlas de manera diferenciada, pues la norma general de dos años de duración podría estar sobreestimada en algunos casos y subestimada en otros. En realidad, podría estar sobreestimada en todos los casos. La disminución de la duración de la formación técnica a dos años no significó una merma en la competitividad laboral de los egresados, por lo que cabe preguntarse si una nueva disminución, a un año por ejemplo, podría acogerse sin riesgos al menos para algunas de las actuales especialidades en oferta (Secretariado, Ventas, entre otras). Por otra parte, convendría limitar la oferta de especialidades técnicas a aquellas que son efectivamente reconocidas por el mercado laboral. En este sentido, es cuestionable la oferta de todo el sector de Administración. El hecho de que una proporción muy significativa de egresados TP trabajen en sectores distintos al de su especialidad, constituye un argumento a favor de las medidas anteriores, en el sentido que confirman que el contenido de la formación técnico-profesional es más bien irrelevante para el destino laboral de los jóvenes, salvo excepciones. Este estudio sugiere que más relevante que la formación es la voluntad de trabajo de los jóvenes y la adopción temprana de una identidad laboral.

De manera paradojal, la contribución de la formación técnico-profesional a las políticas de promoción social radica en su limitación y no en su expansión. En el contexto nacional actual de expansión de educación superior, la educación media técnico-profesional puede aportar en la medida en que se focalice en especialidades que el mercado efectivamente reconozca y en la medida en que no limite las oportunidades de los jóvenes para acceder a la educación superior, como es actualmente el caso. Para ello, los estudiantes que elijan una formación técnica deben tener no menos, sino más oportunidades de formación general que los habilite para seguir estudios superiores, al menos las mismas oportunidades que sus pares de la formación humanista-científica. 


\section{REFERENCIAS BIBLIOGRÁFICAS}

Arzola, S., Collarte, C., Cornejo, J., Etchegaray, F. y Franklin, J. (1993). Destino educativo laboral de los egresados de la Enseñanza Media. Santiago: Ministerio de Educación.

Bravo, D., Cáceres, C., J. Castillo, J., Montero, P. y Saint-Jean, D. (1998). Evaluación del marco institucional de la Educación Media Técnico Profesional. Santiago: Ministerio de Educación.

Centro de Investigación y Desarrollo de la Educación (2008). VII Encuesta CIDE a actores del sistema educativo. Informe final. Santiago: Universidad Alberto Hurtado.

Centro de Investigación y Desarrollo de la Educación (2009). Estudio sobre la implementación curricular de la educación media técnico profesional: informe final. Santiago: Ministerio de Educación.

Contreras, D., Cooper, R., Herman, J. y Neilsen, Ch. (2004). Dinámica de la pobreza y movilidad social: Chile 1996-2001. Santiago: Universidad de Chile, Depto. de Economía.

Colegio de Profesores (2007): Un primer acercamiento a la relegada Enseñanza Media Técnico Profesional. Revista Docencia $\mathrm{N}^{\circ}$ 32, 34-43.

Consejo Asesor Presidencial para la Calidad de la Educación (2006). Informe Final. Santiago.

Cox, C. (2003). Las políticas educacionales en Chile en las últimas dos décadas del siglo XX. En C. Cox, Políticas Educacionales en el cambio de siglo (19-113). Santiago: Editorial Universitaria.

Espinosa, O. (2008): La implementación de la reforma curricular en la educación media técnicoprofesional. Informe Final. Santiago: Ministerio de Educación (FONIDE).

Jakubowski, M., Patrinos, H. \& Porta, E. (2010). The impact of the 1999 education reform in Poland. Education Working Paper $\mathrm{N}^{\circ} 49$, Paris, OCDE.

Marinakis, A. (2004). La rigidez de los salarios en Chile. Santiago: Oficina Internacional del Trabajo.

Marinakis, A. (2009): Chile, fijación del salario mínimo en período de crisis. Global Job Crisis Observatory, OIT. En www.ilo.org/pls/apex (consultado en octubre 2009).

Ministerio de Educación (2006): Más y mejores técnicos para Chile. Estrategia Bicentenario. Santiago.

Ministerio de Educación (2008): Estadísticas de la Educación 2007.

Miranda, M. (2003): Transformación de la Educación Media Técnico Profesional. En C. Cox, Políticas Educacionales en el cambio de siglo (375-417). Santiago: Editorial Universitaria.

OCDE (2004). Revisión de Políticas Nacionales de Educación, Chile. Santiago, editado por el Centro para la Cooperación con los países no miembros de la OCDE y el Ministerio de Educación.

OCDE-Banco Mundial (2009). La Educación Superior en Chile. Revisión de Políticas Nacionales de Educación. Santiago, Ministerio de Educación.

Ortiz, I. (2009). ¿Es relevante la educación media técnico-profesional? Persona y Sociedad vol. XXIII, n. 3, 99-115.

Sánchez, S. y Escudero, C. (2008): Trayectorias educacionales y laborales de los técnicos en Chile. Revista Calidad en la Educación, n. 29, 18-34.

Sepúlveda, L. (2009). Estado y perspectivas de la enseñanza media técnico profesional en Chile: un estudio sobre las orientaciones estratégicas predominantes en los actores. Informe final. Santiago: Ministerio de Educación (FONIDE).

Servat, B. (2007). Efectividad de la Enseñanza Media técnico-profesional. Destino promoción 2004, Región Metropolitana. Santiago: Pontificia Universidad Católica. (Informe de investigación presentado en el Encuentro Nacional de Investigación en Educación 2007, organizado por el CPEIP).

Sevilla, M.P. y Miranda, L. (2009): Estudio de seguimiento de egresados de la enseñanza media técnico-profesional. Santiago: Universidad de Chile, Depto. de Economía.

Vargas, S., Henríquez, A., Aravena, J. y E. Ogrodnik (2006): Estudio inserción laboral de egresados de la Educación Media Técnico-Profesional y de la Educación Técnica de Nivel Superior en el sector de la Administración y Comercio de la Región Metropolitana: informe final. Santiago: Programa Chile-califica. 